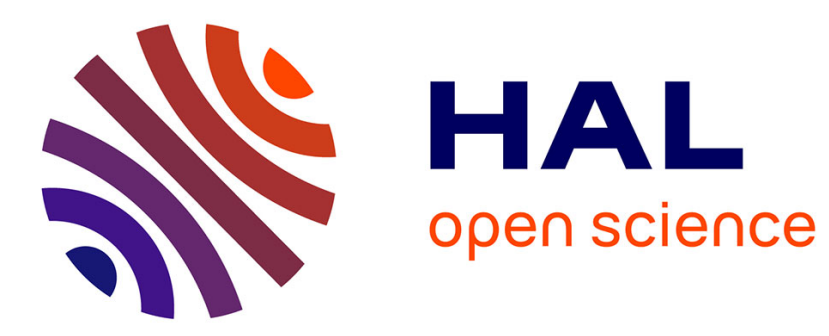

\title{
Performances and Stability Analysis of a novel 8T1R Non-volatile SRAM (NVSRAM) versus Variability
}

\author{
Hussein Bazzi, Hassen Aziza, Mathieu Moreau, Adnan Harb
}

\section{To cite this version:}

Hussein Bazzi, Hassen Aziza, Mathieu Moreau, Adnan Harb. Performances and Stability Analysis of a novel 8T1R Non-volatile SRAM (NVSRAM) versus Variability. Journal of Electronic Testing: Theory and Applications, 2021, 37 (4), pp.515-532. 10.1007/s10836-021-05965-x . hal-03501909

\section{HAL Id: hal-03501909 \\ https://hal.science/hal-03501909}

Submitted on 23 Dec 2021

HAL is a multi-disciplinary open access archive for the deposit and dissemination of scientific research documents, whether they are published or not. The documents may come from teaching and research institutions in France or abroad, or from public or private research centers.
L'archive ouverte pluridisciplinaire HAL, est destinée au dépôt et à la diffusion de documents scientifiques de niveau recherche, publiés ou non, émanant des établissements d'enseignement et de recherche français ou étrangers, des laboratoires publics ou privés. 


\title{
Performances and Stability Analysis of a novel 8T1R Non-volatile SRAM (NVSRAM) versus Variability
}

\author{
Hussein Bazzi ${ }^{1,2}$, Hassen Aziza ${ }^{1}$, Mathieu Moreau ${ }^{1}$, Adnan Harb ${ }^{3}$, Senior Member, IEEE \\ ${ }^{1}$ IM2NP-UMR CNRS 7334, Aix-Marseille University, Marseille, France \\ ${ }^{2}$ Department of Electrical and Electronics Engineering, Lebanese International University, Beirut, Lebanon \\ ${ }^{3}$ Department of Electrical and Electronics Engineering, The International University of Beirut, Beirut, Lebanon \\ hussein.bazzi36@gmail.com, hassen.aziza@univ-amu.fr, mathieu.moreau@univ-amu.fr, adnan.harb@liu.edu.lb
}

\begin{abstract}
Static Random-Access Memories (SRAMs) are an integral part of the chip industry, occupying a noticeable share of the memory market due to their high performance and compatibility with CMOS technology. Traditional SRAMs do not have the capacity to retain data after power-off, preventing their use in non-volatile applications. This paper presents a novel Non-Volatile SRAM (NVSRAM) device based on Resistive RAM (RRAM) technology. A comparison between SRAM and the proposed NVSRAM performances is proposed at both cell and memory array level. The comparison covers several metrics such as energy consumption, area and static noise margin (SNM). Moreover, this work proposes a deep analysis of the impact of RRAM variability as well as the CMOS subsystem variability on the NVSRAM performances. The proposed structure demonstrates robust NVSRAM performances in terms of stability and reliability despite RRAM variability.
\end{abstract}

Index Terms - Static Random-Access Memories (SRAMs), Non-Volatile SRAM (NVSRAM), Resistive RAM (RRAM), energy, area, variability, switching time, Static Noise Margin (SNM).

\section{INTRODUCTION}

For modern high-performance technologies, the memory subsystem directly affects the performance of computing systems [1]. The memory subsystem includes Static RandomAccess Memory (SRAM), Dynamic Random-Access Memory (DRAM), and Flash memories. All these memories are charge-based. In SRAM, the data are stored as charges at the nodes of the cross-coupled inverters [2, 3]. SRAM has been widely used in the memory market. This rapid growth is backed by SRAM appealing characteristics, including fast operations and low power consumption in standby mode [4]. Moreover, SRAM cell uses the same fabrication process as logic, thus no extra cost is needed. These properties are unique for SRAM when compared to other memories such as DRAM or Flash memories. However, as the technology scales down, SRAM standby leakage power is becoming one of the most critical concerns for low power applications [5], particularly when battery-powered applications are targeted [6]. Therefore, there is a need for energy efficient nonvolatile SRAM cell architectures able to retain data after power-off: data are moved to the non-volatile memory elements to retain the system state after power interruption and restored after power-ON.
Today's dominant Non Volatile Memory technology is Flash. But its endurance is limited (approximately $10^{6}$ cycles), and its operation access frequency is very slow, with program/erase time in the order of microseconds to milliseconds [7]. Moreover, Flash devices need high voltages for storage operations which is hardly compatible with ultralow power applications. The quest for low power NVM solutions is the main driving force behind the rapid growth of emerging memories, such as Ferroelectric RAM (FeRAM) [8], Spin Torque Transfer RAM (STT-RAM) [9], Phasechange RAM (PCRAM) [10] and Resistive RAM (RRAM) which encompass Conductive Bridge RAM (CBRAM) and Oxide based-RAM Memories (OxRAMs) [11,12]. RRAM is considered as a promising solution that can elevate SRAM to the next level in the memory technology hierarchy [13]. SRAM can be combined with Resistive RAM (RRAM) technology to create a new hybrid structure referred to as Non-Volatile SRAM (NVSRAM) [14]. Indeed, due to its relatively small write and read latency and high density [15], RRAM is considered as a promising candidate for designing NVSRAMs memory circuits. The latter is expected to replace traditional SRAM in SoC applications [16]. However, from a reliability point of view, RRAM is affected by variability. Indeed, the variance from cycle to cycle $(\mathrm{C} 2 \mathrm{C})$ and from device to device (D2D) can be very large, impacting directly the memory cell performances and particularly the resistance $\mathrm{ON} / \mathrm{OFF}$ ratio. Although several NVSRAM architectures have appeared in the literature [17-25], none of the related work has validated the stability and performances of the proposed NVSRAM memory cells versus RRAM variability as well as CMOS variability. An attempt to address this issue was proposed in [26], but the FORMING operation of the RRAM memory cell is missing as well as the use of a RRAM model calibrated on actual RRAM devices for simulations. Moreover, the impact of the RRAM variability on Static Noise Margins (SNMs) is not analysed and the variability of the CMOS subsystem is not accounted. Also, projections at the circuit level are missing.

In this paper, we address these issues by leveraging on an OxRAM model calibrated on silicon [27] and featuring a FORMING operation as well as a variability dependency in agreement with experimental measurements [28]. This work is motivated by the strong variability inherent to actual RRAM technologies and more particularly to the OxRAM 
technology addressed in this study. The variability analysis also targets the CMOS subsystem (including transistor mismatch $[29,30])$ and especially the memory cell access transistor as its impact on the memory cell electrical characteristics is dominant $[31,32]$. This type of analysis combining OxRAM and CMOS variability is completely missing in the current state-of-the art. In this context, the contributions of this paper are as follows:

1) A novel NVSRAM memory cell architecture implemented in a $130-\mathrm{nm}$ high voltage CMOS technology from STMicroelectronics [33] is introduced.

2) A deep analysis regarding the effect of variability (including OxRAM and CMOS variability) is conducted based an OxRAM model calibrated on silicon.

3) A detailed comparison between traditional SRAMs and the proposed NVSRAM is conducted at the cell level as well as the circuit level.

4) Factor of merits including energy consumption, switching times during FORMING, RESET and SET operations, SNMs and area are extracted and analysed.

In section II, OxRAM technology is introduced and OxRAM variability is investigated. In section III, the design of the proposed NVSRAM is presented and compared with a traditional SRAM cell at the cell level. In section IV, projections at the memory array level are provided. In Section V, SRAM and NVSRAM performances are compared based on electrical simulations. In section VI, the impact of variability is investigated based on Monte Carlo (MC) simulations. Section VII presents some concluding remarks.

\section{OXRAM TECHNOLOGY}

\section{A. Background}

An OxRAM device consists of three layers including two metal electrodes and an insulator layer [34], structure known as Metal-Insulator-Metal (MIM) shown in Fig. 1(a). This device can be integrated in the Back End Of Line (BEOL) of CMOS technology as depicted in Fig. 1(b), presenting important benefits in term of area and data transfer latency.

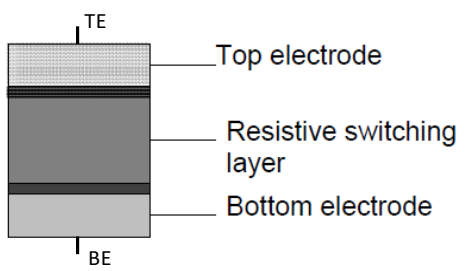

(a)

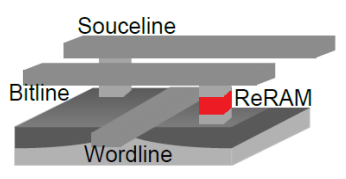

(b)
Fig. 1. (a) Basic RRAM structure and (b) its integration in the BEOL

Fig. 2 (a) and (b) show OxRAM I-V characteristic in linear and logarithmic scale respectively, extracted from actual OxRAM devices [27]. The first step in the OxRAM programming operation is FORMING where a high voltage is applied across the memory cell to switch the cell from HRS (high resistive state) to LRS (low resistive state). FORMING operation is performed once in the OxRAM device life. After FORMING, SET and RESET operations are executed by applying specific voltages on the electrodes of OxRAM cell (i.e. Vset and VRESET) [35]. Based on the linear curve presented in Fig. 2 (a), the VSET value needed to switch to LRS state is equal to $0.57 \mathrm{~V}$, while the $\mathrm{V}_{\text {RESET }}$ value required to switch back to HRS state is equal to $-0.7 \mathrm{~V}[36,37]$. Note that $\log$ curve (Fig. 2 (b)) is the classical representation of the OxRAM I-V hysteresis as it amplifies low current values.

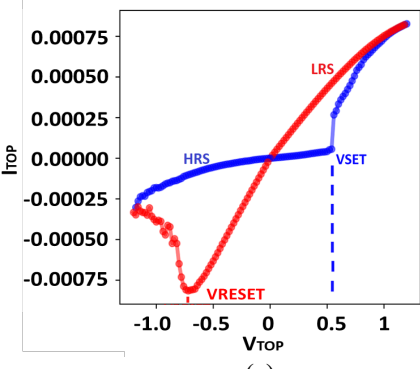

(a)

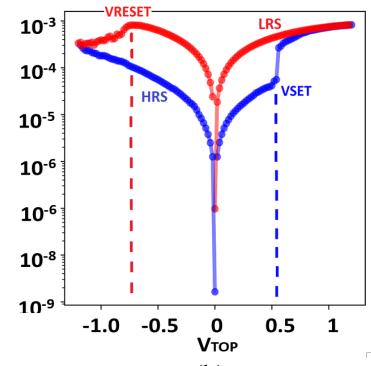

(b)
Fig. 2. I-V characteristic of an OxRAM cell in (a) linear and (b) log scale

\section{B. OxRAM Model}

The OxRAM model approach used in this work (IM2NP model [27]) is based on the formation and destruction of oxygen vacancies by the induced electric field inside the insulator layer. The model is calibrated on silicon $(130 \mathrm{~nm}$ technology from STMicroelectronics) with no convergence issues when used in combination with CMOS technology. Also, it is considered as one of the few models considering the FORMING operation, which is a crucial factor when targeting the fabrication of the devices. Moreover, the 130 $\mathrm{nm}$ technology provides High Voltage (HV) transistors needed for the FORMING operation.

As the OxRAM cells are integrated in the BEOL (on top of CMOS transistors), the performances of the proposed NVSRAM cell rely on the CMOS subsystem. If the performance in terms of speed and energy consumption is improved for advanced CMOS technology nodes (which is usually the case), it would be reflected in the NVSRAM performance. Regarding the area, it should be drastically reduced as the footprint of the transistors is reduced.

Fig. 3 depicts I-V characteristics for (a) SET, RESET and (b) FORMING operations measured on a large number of memory elements coming from [38-39]. A standard deviation of about $\pm 5 \%$ on selected parameters ( $\alpha$ : composition and $L_{x}$ : local variations of thickness) enables satisfactorily accounting for the variability [27]. A modulation of $\alpha$ impacts the creation/destruction kinetics of the conductive filaments (CFs) which introduces a variability in the programming voltage ( $\left.V_{\text {SET }} \& \mathrm{~V}_{\text {RESET }}\right)$ and in the CF width. On the other hand, $L_{x}$ variation impacts the current though the virgin oxide (i.e., post-FORMING current). The simulation data closely matches the measurement data as shown in Fig. 3, certifying the reliability of the model.

\section{OxRAM Variability}

OxRAM devices suffer from a significant variability that can be divided into two categories, D2D and C2C variabilities [40]. The variability is demonstrated experimentally based on an elementary memory array. Fig. 4 
(a) presents a classical 1T1R array which is used for experimental measurements. Memory cells are grouped to form eight 8-bit memory words. Word Lines $\left(W L_{X}\right)$ are used to select the active row, Bit Lines $\left(B L_{X}\right)$ are used to select active columns during a SET operation and Source Lines $\left(S L_{X}\right)$ are used to RESET a whole memory word or an addressed cell. Fig. 4 (b) presents the layout view of the memory array. Due to the limited pin out of the probe card used in the experimental phase, only a $7 \times 7$ memory array is available for our experiments (subset of the 8x8 array). Based on this elementary memory array, OxRAM variability is examined.

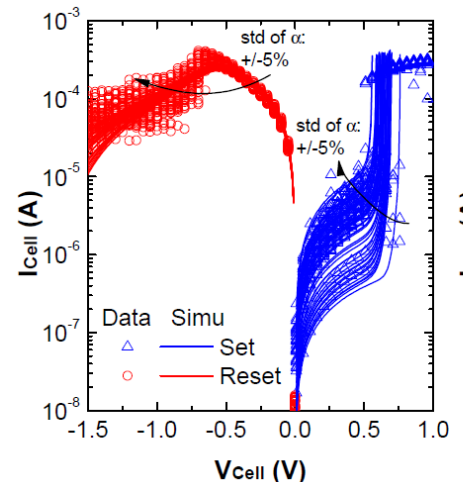

(a)

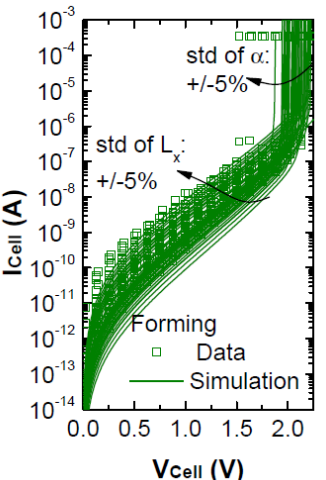

(b)
Fig. 3. Measured and corresponding simulated I-V characteristic showing LHS and HRS variations and switching parameters variation after (a) SET, RESET and (b) FORMING operations [28]

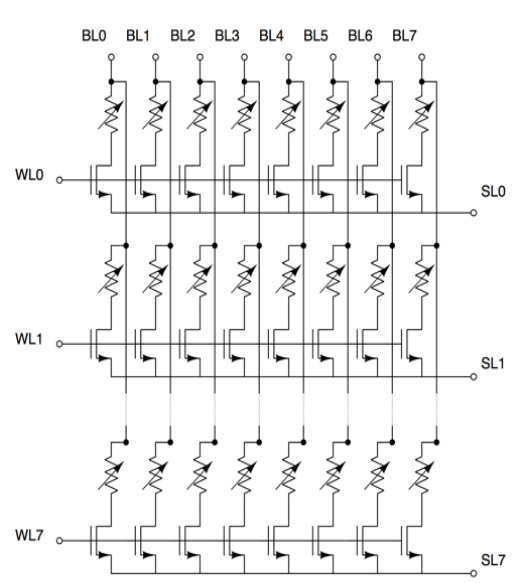

(a)

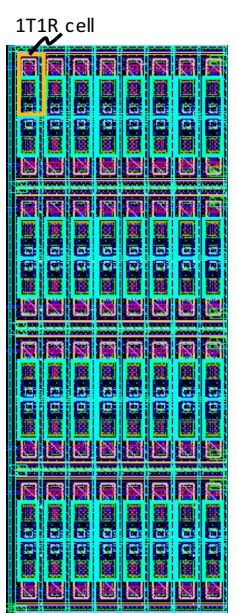

(b)
Fig. 4. (a) 8x8 OxRAM memory array and (b) corresponding layout view

Fig. 5 shows Ron and Roff distributions combining C2C and D2D variabilities. Distributions are the result of 1000 consecutive RESET/SET operations applied to the whole memory array (1000x49 cells). HRS and LRS variability is clearly demonstrated and HRS distribution spread is more pronounced compared to the LRS spread, which is a common feature of the considered OxRAM technology. Hence, this drawback of the technology needs to be considered when designing OxRAM-based NVSRAMs. Indeed, in a NVSRAM context, variability can lead to a weak SET or RESET of the OXRAM when data is moved to the non- volatile memory element. Moreover, the main problem that can occur is to RESTORE a false data after an extreme weak RESET operation (HRS post-RESET state located in the distribution tail). Table I presents the different voltage levels applied to the OxRAM cell during FORMING, RESET and SET operations. The whole experimental setup is based on a Keysight B1500 semiconductor parameter analyzer [41].

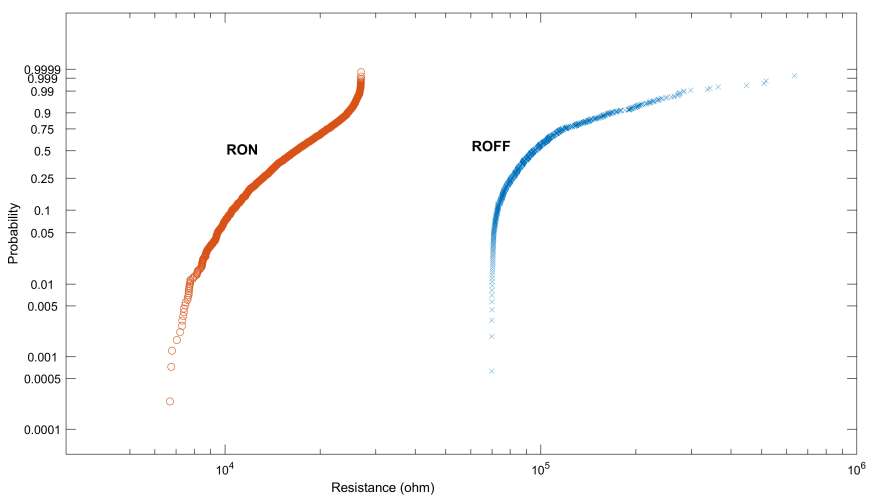

Fig. 5. $\mathrm{R}_{\mathrm{ON}}$ and $\mathrm{R}_{\mathrm{OFF}}$ distribution

TABLE I. STANDARD OPERATING VOLTAGES (CELL LEVEL)

\begin{tabular}{lcccc}
\hline \hline & FORMING & RESET & SET & READ \\
\hline $\mathrm{WL}$ & $2 \mathrm{~V}$ & $4.5 \mathrm{~V}$ & $2 \mathrm{~V}$ & $4 \mathrm{~V}$ \\
$\mathrm{BL}$ & $2 \mathrm{~V}$ & $0 \mathrm{~V}$ & $1.2 \mathrm{~V}$ & $0.2 \mathrm{~V}$ \\
$\mathrm{SL}$ & $0 \mathrm{~V}$ & $1.8 \mathrm{~V}$ & $0 \mathrm{~V}$ & $0 \mathrm{~V}$ \\
\hline \hline
\end{tabular}

\section{CELl LEVEL : SRAM \& NVSRAM}

A NVSRAM cell is formed by the combination of a SRAM cell and an OxRAM memory device where some SRAM nodes are connected to the OxRAM cells. This structure allows backing up data from the SRAM to the OxRAM in the standby mode while the SRAM classical operations (i.e. HOLD, WRITE and READ operations) are maintained in the normal operating mode.

\section{A. 6T SRAM memory cell}

The 6T SRAM memory cell is the classical SRAM structure based on the cross coupled inverters presented in Fig. 6. HOLD, READ and WRITE are the main operations in an SRAM [42]. These operations are performed through Word Lines (WLs) and Bit Lines (BLs). WLs are connected to the gates of the select transistors (M3 and M4), and BLs are connected to the select transistor sources/drains. During the HOLD stage, BLs are disconnected from the SRAM core by deactivating the WLs. Data nodes $\mathrm{Q}_{\text {and }} \mathrm{Q}_{\mathrm{b}}$ retain the data. READ and WRITE operations are completed by activating WL to sense or update the stored data respectively. Fig. 7 shows the 6T SRAM cell layout implemented in a $130 \mathrm{~nm}$ technology, with an area occupancy equals to $34 \mu \mathrm{m}^{2}$. 


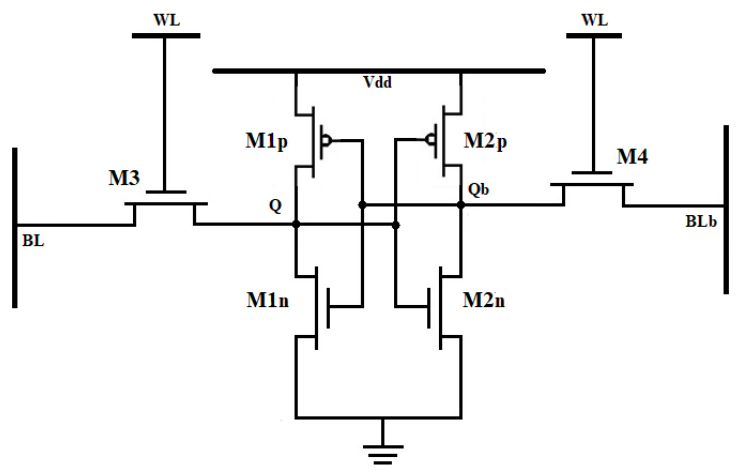

Fig. 6. 6T SRAM cell

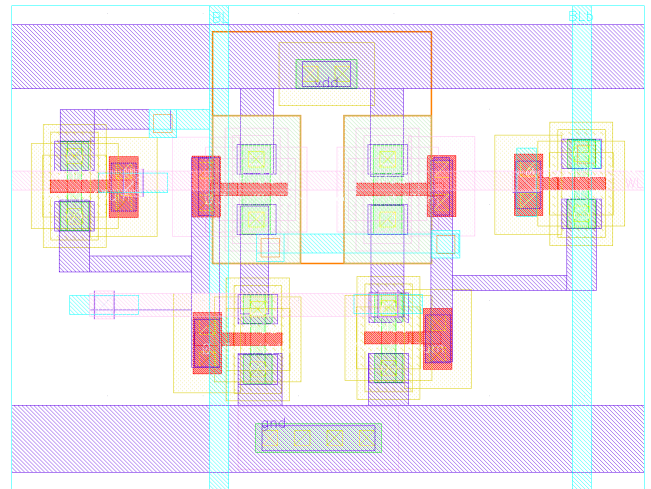

Fig. 7. 6T SRAM Cell layout

\section{B. 8T1R NVSRAM memory cell}

The proposed 8T1R structure is depicted in Fig. 8. The sizing of the transistors is as follow: $\mathrm{M} 1 \mathrm{n}$ and $\mathrm{M} 2 \mathrm{n}(\mathrm{w}=150$ $\mathrm{nm}), \mathrm{M} 1 \mathrm{p}$ and M2p (w=450 nm). M3, M4, M5 and M6 (900 $\mathrm{nm})$. Note that the length of all transistors is fixed $(130 \mathrm{~nm})$. The top electrode (TE) of the OxRAM device is connected to transistor M5 and the bottom electrode (BE) to the node $\mathrm{Q}_{\mathrm{b}}$. In this structure, transistors (M3 and M4) represent the access transistors and transistors (M5 and M6) are used for the nonvolatile storage operations referred to as STORE and RESTORE. STORE consists of two operations: OxRAM SET (stores "1") and OxRAM RESET (stores "0").

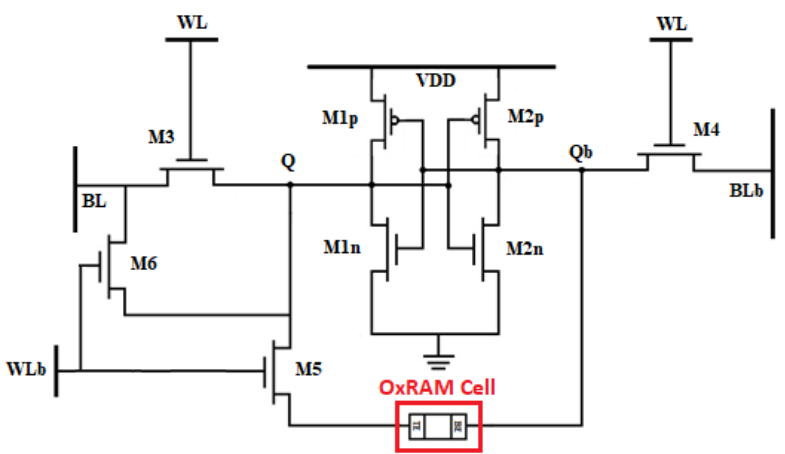

Fig. 8. 8T1R NVSRAM Cell
Before any STORE/RESTORE operation, the OxRAM cell needs to be formed. Forming is achieved in one step where $\mathrm{WL}$ and $\mathrm{BL} b$ are set low and $\mathrm{BL}$ and $\mathrm{WL} b$ are set high, allowing a direct connection between BL and the OxRAM though transistors M5 and M6. BL is set to $5 \mathrm{~V}$ while $\mathrm{BLb}$ is grounded and the OxRAM access transistors (M5 and M6) are controlled by applying a pulse to WLb. After Forming, the STORE operation has to be preceded by a WRITE operation. In this regard, WRITE "0" comes before STORE "0" (RESET) and WRITE "1" comes before STORE "1" (SET). During WRITE "0" operation, WL and BLb are set high, BL and $\mathrm{WL}_{b}$ are set low. On the other hand, BL is set high and $\mathrm{BL} b$ is set low during WRITE " 1 " operation. During STORE, $\mathrm{WL}_{b}$ is set high and WL is set low to access the OxRAM cell for programming: $\mathrm{BL}$ is set low and $\mathrm{BL} b$ is set high during STORE "0" (RESET), besides BL is set high and BLb is set low during STORE "1" (SET). Note that STORE and WRITE operations are independent as WRITE operations (normal operation) are executed separately from the STORE operations (RRAM programming).

Before a READ operation, a Precharge of the BLs is needed where $\mathrm{BL}$ and $\mathrm{BL}_{\mathrm{b}}$ are set to $\mathrm{V}_{\mathrm{DD}}(1.8 \mathrm{~V})$. Then, WL is set high to READ the cell. After STORE (OxRAM SET or RESET), $V_{D D}$ is turned OFF. During RESTORE, $V_{D D}$ is activated and $\mathrm{WL}_{b}$ is set high (all the other signals are kept low) to recover the stored data. Fig. 9 shows the 8T1R NVSRAM cell layout with an area equals to $53 \mu \mathrm{m}^{2}$. This novel memory cell is characterized by its low number of control signals compared to NVSRAMs proposed in [43-45, 20].

Table II illustrates the advantages and drawbacks of different NVSRAMs proposed in the literature in terms of STORE/RESTORE time, RESTORE method, non-volatility, STORE/RESTORE energy, and silicon verification. There are 2 types of RESTORE methods: single-ended and differential (diff.). In the single-ended topology, one OxRAM cell is used, where in the differential topology, two OxRAM cells are used. Another factor is the type of NVSRAM nonvolatility.

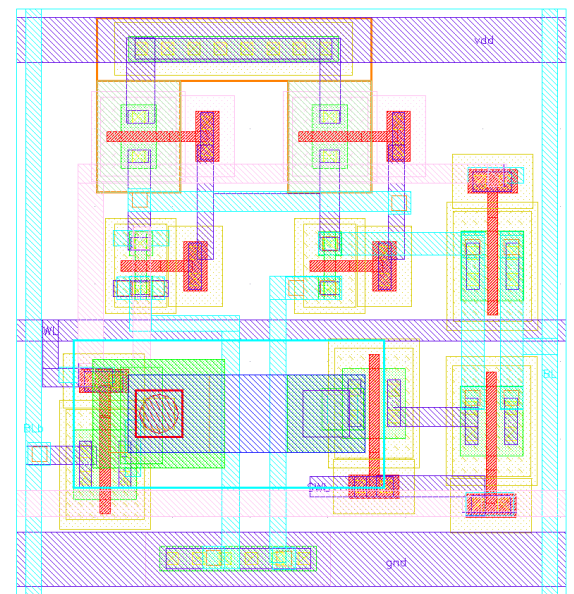

Fig. 9. 8T1R NVSRAM Cell layout 
TABLE II. COMPARISON AGAINST DIFFERENT NVSRAM DESIGNS

\begin{tabular}{|c|c|c|c|c|c|c|}
\hline & $\begin{array}{c}4 T 2 R \\
{[19]} \\
\end{array}$ & $\begin{array}{c}\text { 7T1R } \\
{[20]} \\
\end{array}$ & $\begin{array}{c}8 T 2 R \\
{[43]} \\
\end{array}$ & $\begin{array}{c}7 \mathrm{TT2R} \\
{[44]} \\
\end{array}$ & $\begin{array}{c}8 \mathrm{TT2R} \\
{[45]}\end{array}$ & 8T1R \\
\hline STORE Time & $1.45 \mathrm{~ns}$ & $3 \mathrm{~ns}$ & $3 \mathrm{~ns}$ & $12.6 \mathrm{~ns}$ & $10 \mathrm{~ns}$ & $6.8 \mathrm{~ns}$ \\
\hline $\begin{array}{c}\text { RESTORE } \\
\text { Time }\end{array}$ & $20 \mathrm{ps}$ & $0.22 \mathrm{~ns}$ & $0.22 \mathrm{~ns}$ & $0.37 \mathrm{~ns}$ & $0.36 \mathrm{~ns}$ & $0.45 \mathrm{~ns}$ \\
\hline $\begin{array}{c}\text { RESTORE } \\
\text { Method }\end{array}$ & Diff. & $\begin{array}{l}\text { Single- } \\
\text { ended }\end{array}$ & Diff. & Diff. & Diff. & $\begin{array}{l}\text { Single- } \\
\text { ended }\end{array}$ \\
\hline Non-Volatility & $\begin{array}{l}\text { Real- } \\
\text { time }\end{array}$ & Last-bit & Last-bit & Last-bit & Last-bit & Last-bit \\
\hline $\begin{array}{l}\text { STORE } \\
\text { Energy }\end{array}$ & $594 \mathrm{pJ}$ & $36 \mathrm{pJ}$ & $121 \mathrm{pJ}$ & $218 \mathrm{pJ}$ & 194 pJ & $176 \mathrm{pJ}$ \\
\hline $\begin{array}{c}\text { RESTORE } \\
\text { Energy }\end{array}$ & $30 \mathrm{fJ}$ & $50 \mathrm{fJ}$ & $80 \mathrm{fJ}$ & $0.25 \mathrm{fJ}$ & $0.52 \mathrm{fJ}$ & $81 \mathrm{fJ}$ \\
\hline $\begin{array}{c}\text { FORMING } \\
\text { Energy }\end{array}$ & $1.7 \mathrm{~nJ}$ & $0.35 \mathrm{~nJ}$ & $0.77 \mathrm{~nJ}$ & $1 \mathrm{~nJ}$ & $\begin{array}{c}6.345 \\
\text { nJ }\end{array}$ & $2.5 \mathrm{~nJ}$ \\
\hline $\begin{array}{l}\text { Silicon } \\
\text { Verified }\end{array}$ & No & No & No & Yes & Yes & No \\
\hline
\end{tabular}

For example, some NVSRAM cells offer real-time nonvolatility where the data is stored after every WRITE operation but at the expense of higher power consumption (Real-time). On the contrary, other NVSRAM cells follow the last-bit non-volatility where the data is stored just before shutdown (Last-bit).

STORE energy (hundreds of $\mathrm{pJ}$ for most of the NVSRAM cells) is the result of a programming operation. Whereas, the RESTORE operation is a sensing operation requiring less energy (order of tens $\mathrm{pJ}$ ) and shorter READ pulses. Minimal STORE times are in the range of 1-10 ns (6.8 ns for the proposed $8 \mathrm{~T} 1 \mathrm{R}$ cell) and minimal reported RESTORE times are less than $1 \mathrm{~ns}(0.45 \mathrm{~ns}$ for the proposed $8 \mathrm{~T} 1 \mathrm{R}$ cell). Timing and energy requirements are dictated by the targeted application. When dealing with ultra-low power applications, timing is not an issue (i.e., can be relaxed) but STORE/RESTORE energies need to be lowered. Moreover, the number of STORE/RESTORE operations following power interruptions is application dependent. If power interruptions are frequent, a good balance between timing and energy can be required. Alternatively, parameters such as area and NVSRAM cell complexity (number of control signals and RESTORE method) play an important role when low-cost applications are targeted.

\section{MEMORY ARRAy LEVEL}

In this section, a conventional SRAM memory array is designed and compared with our proposed NVSRAM array. Both arrays follow the same architecture. However, the NVSRAM array requires some additional blocks to complete the non-volatile operations.

\section{A. SRAM Array Design}

In a SRAM array, SRAM cells are arranged to share connections in horizontal rows and vertical columns. In this work, an elementary $4 \times 8$ bytes memory array presented in Fig. 10 is proposed with a total size of 256 cells. The horizontal lines are the WLs, while the vertical lines along which data flow into and out of cells are the BLs. To access a cell for READ and WRITE operations, a specific row and column are selected [46]. The memory array is associated with peripheral circuits as illustrated in Fig. 10. Word decoder and column decoder select WL and BL consecutively, based on the provided address. Note that the column decoder controls numerous peripheral circuits including precharge circuits, column multiplexers, sense amplifiers and write drivers. Write drivers deliver the logic states to an addressed memory word. The BLs are connected to the precharge circuits. Also, BLs are connected to the write drivers and to the sense amplifiers to complete WRITE and READ operations, respectively.

During WRITE operation, data inputs are fed directly to the write drivers. The row decoder controls the selection of WLs, thus addressing the targeted row. The column decoder is used to select a group of columns, where the programing operations are executed. The column multiplexer role is to connect the sense amplifier to the BLs and disconnect the precharge circuit and write driver circuit to ensure that READ operation is completed properly. Before READ, the precharge circuit increases $\mathrm{BL}$ voltage to $\mathrm{V}_{\mathrm{DD}}$ or $\mathrm{V}_{\mathrm{DD}} / 2$. READ operation is initiated by enabling SE signal [47]. Fig. 11 presents the $4 \times 8$ bytes SRAM array layout.

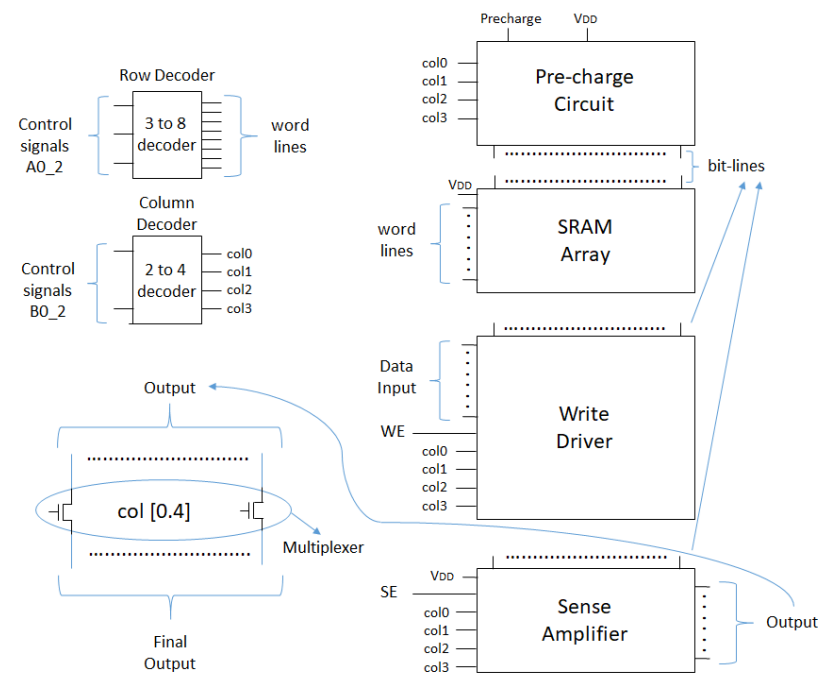

Fig. 10. SRAM Array Design

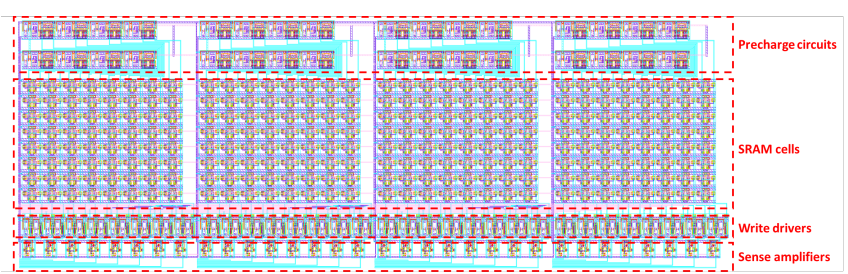

Fig. 11. Layout of $4 \times 8$ bytes SRAM array

Fig. 12 represents SRAM Finite State Machine (FSM) diagram. In this diagram, there are four different states (IDLE, Precharge, READ and WRITE), and two control signals (reset and RW). When the state is "IDLE" and "reset $=1$ ", the FSM maintains its current state. "IDLE" state moves to "Precharge" state when "reset $=0$ " and moves back to "IDLE" when "reset $=1$ ". The next state after "Precharge" is 
"READ" or "WRITE", if "RW = $0 "$ and "RW = 1 " respectively. "WRITE" and "READ" states move to "IDLE" state, when "reset $=1$ ". Note that if no control signal is activated, "WRITE" and "READ" states go back to "Precharge". The complete SRAM array structure including the FSM block is shown in Fig. 13 (a). The structure inputs are the clock, the address bus, the data bus, and control signals. Fig. 13 (b) shows SRAM memory map with the address bus LSB and MSB bits.

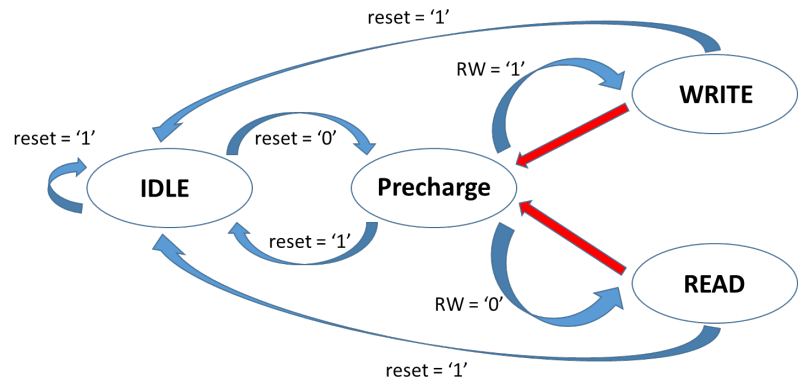

Fig. 12. SRAM Finite State Machine Diagram

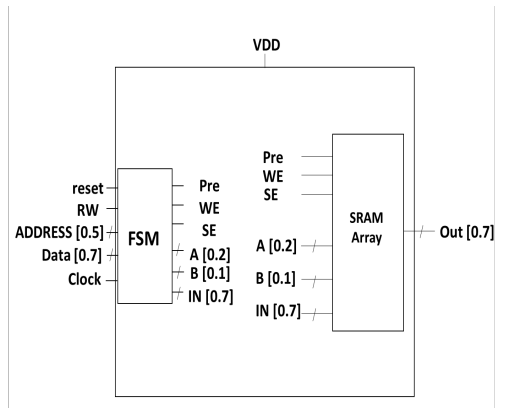

(a)

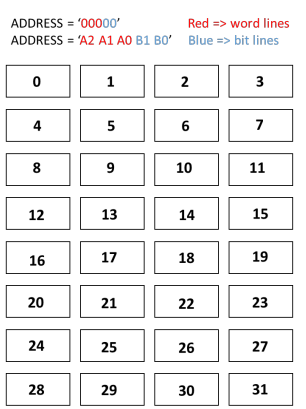

(b)
Fig. 13. (a) Complete SRAM array structure with FSM circuitry (b) SRAM address distribution

\section{B. NVSRAM Array Design}

The proposed NVSRAM array structure is adjusted to the 8T1R cell presented in Fig. 8 which is the core cell of the memory array. The design includes all the peripheral circuits associated with a classical memory array with some marginal modifications as illustrated in Fig. 14. Additional logic circuits are added to handle the STORE (SET \& RESET) and RESTORE operations. Note that, $\mathrm{WL}_{\mathrm{b}}$ has its own decoder to have a direct control over the STORE operations. Fig. 15 presents the $4 \times 8$ bytes NVSRAM array layout.
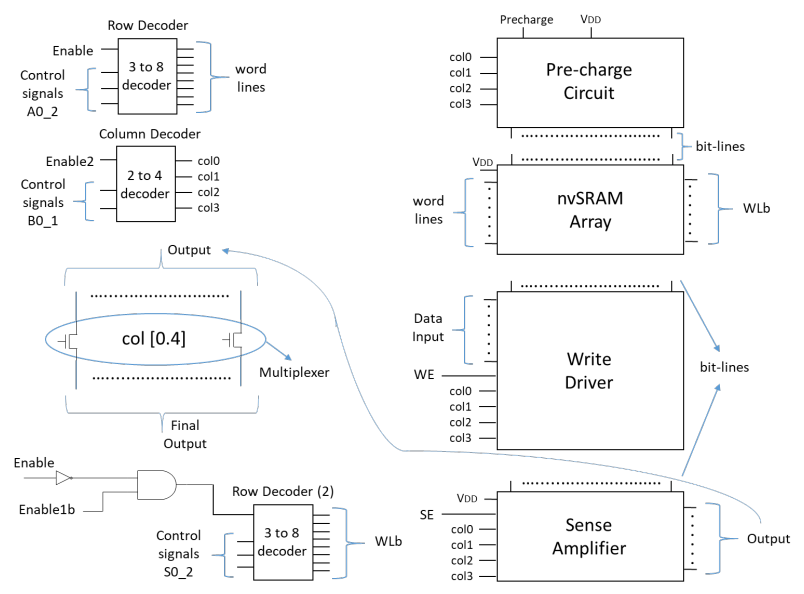

Fig. 14. NVSRAM Array Design

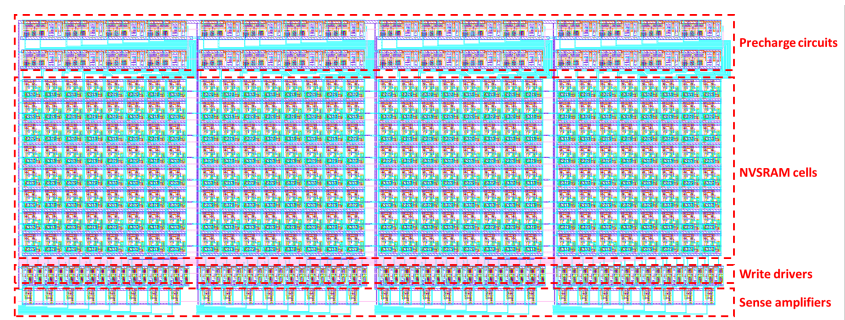

Fig. 15. Layout of $4 \times 8$ bytes NVSRAM array

Fig. 16 represents NVSRAM FSM diagram. In this diagram, three additional states (FORMING, STORE and RESTORE), and two additional control signals (FORMING control (Fc) and STORE control (Sc)) are available compared to the FSM associated with a classical SRAM array. In all, seven states and four internal signals are needed. When the state is "IDLE" and "reset = 1", the FSM maintains its current state. "IDLE" state moves to "Precharge" state when "reset = $0 \& \mathrm{Sc}=1$ ", and to "RESTORE" state when "reset $=0$ \& Sc $=0$ ". Proceeding to "READ" and "WRITE" states, "Fc $=0$ \& $\mathrm{RW}=0$ " and "Fc $=0 \& \mathrm{RW}=1$ ", respectively. To end up in "FORMING" state, "Fc = 1. After "WRITE", the FSM can move to the "STORE" state where "reset $=0 \& \mathrm{Sc}=1$ ". All states move to "IDLE", when "reset $=1$ ". The complete NVSRAM array structure with the added FSM block is shown in Fig. 17 (a). The NVSRAM circuit inputs signals are the clock, the address bus, the data bus, and the FSM control signals. Fig. 17 (b) shows the NVSRAM memory map with the address bus LSB and MSB bits.

\section{SimUlation Results AND COMPARISON}

SRAM and NVSRAM simulations results are proposed in this section at the memory array level. Voltage pulses levels for programming operations are set to be compliant with the available voltage supplies $(1.8 \mathrm{~V}, 3.3 \mathrm{~V}$ and $5 \mathrm{~V})$. 


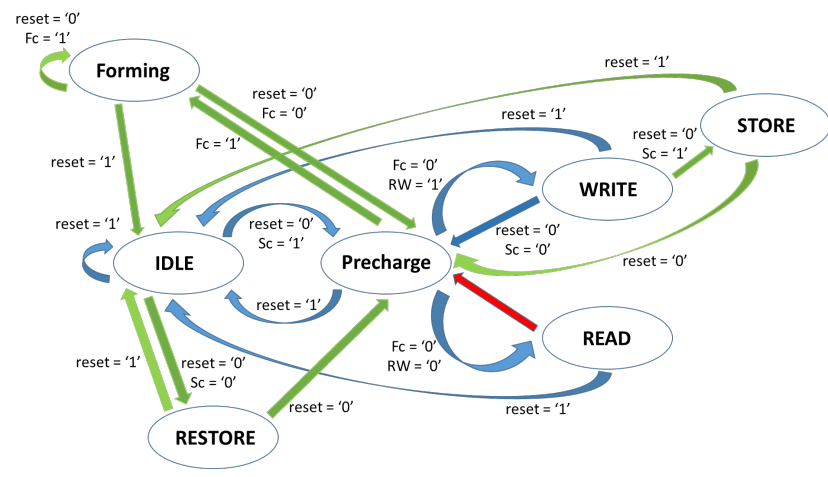

Fig. 16. NVSRAM Finite State Machine Diagram

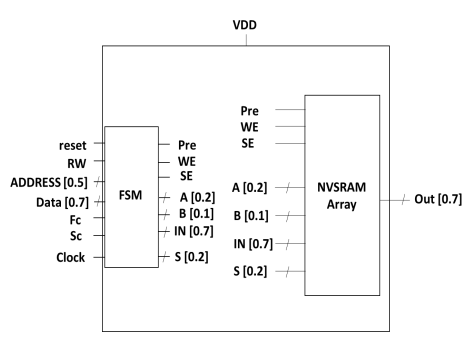

(a)

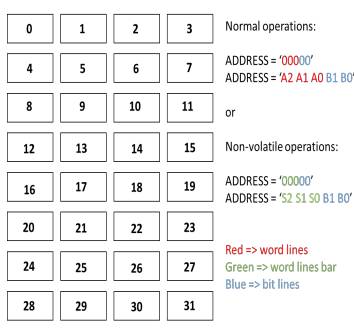

(b)
Fig. 17. (a) Complete NVSRAM array structure with FSM circuitry (b) NVSRAM address distribution

\section{A. SRAM array simulation results}

Based on the simulation of the circuit presented in Fig. 13 (a), the internal signals (FSM outputs) Pre, WE, SE, A, B, and IN have been extracted. These signals are presented in Fig. 18 and Fig. 19. Fig. 18 presents the row and column decoder inputs and outputs during the following set of operations: Precharge - WRITE - HOLD - Precharge READ. When A0 and A1 signals of the row decoder are set high, WL3 is selected. For the column decoder, when B0 is high and B1 is low, coll is selected.

The SRAM array timing waveforms are presented in Fig. 19. The precharge circuit is first activated (Precharge signal is set to "0"). Write drivers and sense amplifiers controlled respectively by WE and SE signals are deactivated. In this stage, the row decoder is OFF, as the memory cells are not selected, and the column decoder is turned ON to select the BLs to be precharged. During WRITE, the precharge circuit is disabled (Precharge is set to " 1 "), and the sense amplifier circuit is OFF ( $\mathrm{SE}=$ " 0 "). Write driver is turned $\mathrm{ON}$ (WE = " 1 "), and the row decoder is enabled to access the addressed cells. During HOLD state, all the signals are turned OFF (except $\mathrm{V}_{\mathrm{DD}}$ ) to maintain the data in the cross coupled inverters. HOLD operation is followed by Precharge to initiate a READ operation. During READ, precharge and write driver circuits are turned OFF. The column decoder connects the BLs to the selected sense amplifiers. The output (out = " $0 ")$ and (out = " $1 ")$ are the result of WRITE " 0 " and WRITE "1" operations, respectively.
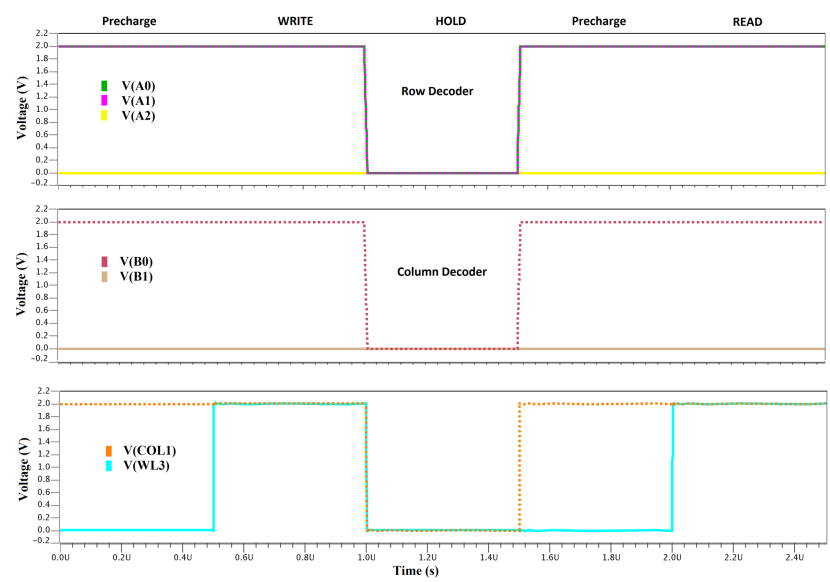

Fig. 18. Decoder Control Signals
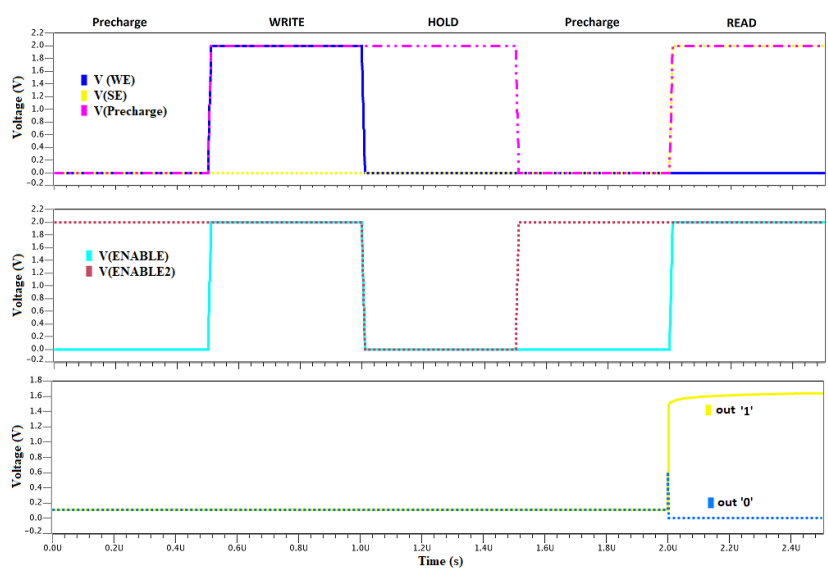

Fig. 19. SRAM array timing waveforms

\section{B. $8 T 1 R$ memory array simulation results}

Based on the simulation of the circuit presented in Fig. 17 (a), Pre, WE, SE, A, B, IN and S have been extracted. These signals are presented in Fig. 20 and Fig. 21. The decoders control signals are presented in Fig. 20. All signals of the row decoder are set high to select WL7 row. For the column decoder, all signals are set high to select col3 column.

The timing waveforms of the NVSRAM array simulation are presented in Fig. 21. Precharge and WRITE "1" operations precede the FORMING stage. During WRITE "1", the precharge circuit and the sense amplifier are OFF (Precharge $=$ " $1 " \& \mathrm{SE}=$ "0"). The write driver is enabled $(\mathrm{WE}=$ " $1 ")$ and the data bus is set to "0xAA". Also, the second row decoder that control $\mathrm{WL}_{b}$ is set ON (ENABLE1b $=$ "1", see Fig. 14). After FORMING, a WRITE operation is executed after Precharge. During STORE operation, most of the peripheral circuits (precharge circuit, write driver, sense amplifier, and row decoder) are disabled. Moreover, the column decoder is kept high (ENABLE2 = " 1 ") and the second row decoder $\left(\mathrm{WL}_{b}\right)$ is enabled (ENABLE $1 \mathrm{~b}=$ " 1 ") to access the OxRAM cell. The next stage is a Precharge followed by a READ operation where the output signal (out) is sensed according to the data bus values (" 0 " or " 1 "). During the "OFF" state $\mathrm{V}_{\mathrm{DD}}$ is set to 0 . After power ON, the 
RESTORE operation is performed to recover the data on nodes Q and $\mathrm{Qb}_{\mathrm{b}}$ of the NVSRAM cell. During this operation, the power supply $V_{D D}$ is enabled allowing the current to pass through the OxRAM cell to the data nodes. After RESTORE, a second Precharge and READ operations are executed to check that the data restored matches the initial data. Regarding the recovery time (time needed to obtain a stable logical value after power-on), according to Fig. 21, its nominal value can be evaluated to $500 \mathrm{~ns}$.
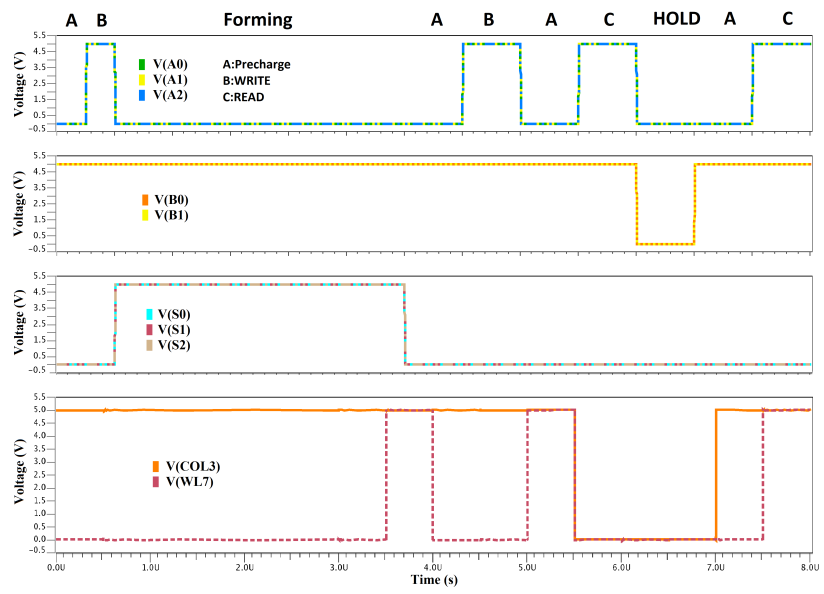

Fig. 20. Decoders Control Signals
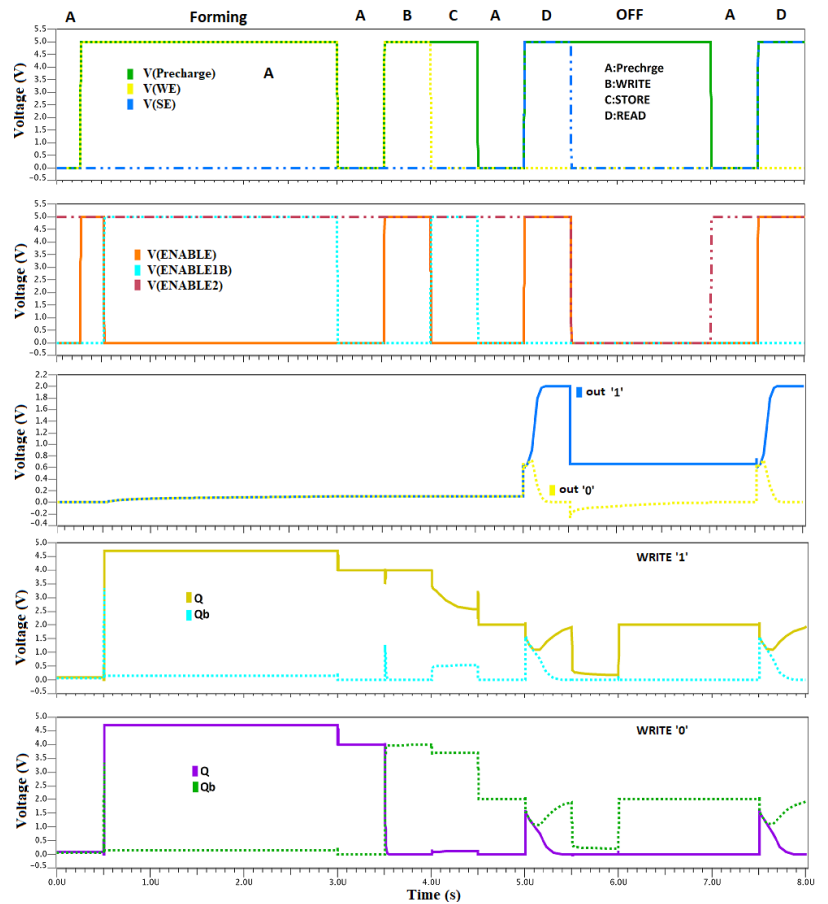

Fig. 21. NVSRAM array timing waveforms

\section{Comparison}

Table III shows a comparison between SRAM and NVSRAM circuits in term of energy consumption, area, switching time, and SNM. The NVSRAM array has a slightly higher energy consumption $(2.6 \%)$ when used in normal operations. The data of the NVSRAM cells is stored in the
OxRAM devices before the "OFF" state. This sequence requires specific programming operations to be completed (STORE before and RESTORE after power down). During HOLD state, $V_{D D}$ is kept high to ensure the data is retained. The energy consumption of both arrays in this state is around $38 \mathrm{pJ}$, since the OxRAM device does not interfere in the normal operations (SRAM mode).

The area of the SRAM and NVSRAM arrays is 18979 $\mu \mathrm{m}^{2}$ and $25775 \mu^{2}$, respectively. Hence, the area of NVSRAM array is $34.9 \%$ larger compared to the SRAM array. The layouts of SRAM and NVSRAM have similar structure since both rely on the same design for the peripheral circuits. However, since the SRAM array has lower number of transistors and does not embed resistive memory elements, its area is much smaller than the one of the NVSRAM array.

SRAM stability is conventionally defined by the SNM [45] which is the maximum value of the DC noise voltage that can be tolerated by the SRAM cell without altering/flipping the stored bit. Stability is the most important criterion in the design of an SRAM as it guarantees that the SRAM cell can hold its data under READ and HOLD conditions. In practice, the smallest square between the voltage transfer characteristics (VTCs) of the two inverters is used to graphically determine the SNM [48]. The nominal SNMs of the considred SRAM cell is $704 \mathrm{mV}, 180 \mathrm{mV}$ and $864 \mathrm{mV}$ during HOLD, READ and WRITE states, respectively. Similiarly, the nominal SNMs of the NVSRAM is $605 \mathrm{mV}, 150 \mathrm{mV}$ and $795 \mathrm{mV}$ during HOLD, READ and WRITE states, respectively. The smaller READ SNM of the NVSRAM cell (16.67\% lower compared to the SRAM) shows that the cell is more vulnerable to data corruption during a READ operation. Write Margin (WM) is defined as the $\mathrm{BL}_{b}$ value when nodes $\mathrm{Q}$ and $\mathrm{Q}_{\mathrm{b}}$ flip. The lower that value is, the harder it is to write the cell, implying a smaller write margin. The WM nominal value is $670 \mathrm{mV}$ for the SRAM and $605 \mathrm{mV}$ for the NVSRAM.

To gain non-volatility, the area is compromised ( $+34.9 \%)$ as well as some performance parameters such as SNMs and WM (8 to $17 \%$ loss). As already mentioned, the choice of moving from a classical SRAM to a NVSRAM is application dependent. For low-cost applications, a $35 \%$ increase in the area can be problematic. However, for ultra-low power applications, the area overhead can be justified.

TABLE III. COMPARISON BETWEEN SRAM AND NVSRAM ARRAYS

\begin{tabular}{lccc}
\hline \hline & SRAM & NVSRAM Difference (\%) \\
\hline $\begin{array}{l}\text { Energy Consumption (Normal } \\
\text { operations) }\end{array}$ & $13.64 \mathrm{~nJ}$ & $14 \mathrm{~nJ}$ & $+2.6 \%$ \\
Energy Consumption (HOLD) & $38.34 \mathrm{pJ}$ & $38.34 \mathrm{pJ}$ & - \\
Area (4x8 bytes) & $18765 \mu \mathrm{m}^{2}$ & $25312 \mu \mathrm{m}^{2}$ & $+34.9 \%$ \\
Nominal SNM (HOLD state) & $704 \mathrm{mV}$ & $605 \mathrm{mV}$ & $-14 \%$ \\
Nominal SNM (READ state) & $180 \mathrm{mV}$ & $150 \mathrm{mV}$ & $-16.67 \%$ \\
Nominal SNM (WRITE state) & $864 \mathrm{mV}$ & $795 \mathrm{mV}$ & $-8 \%$ \\
Write Margin (WM) & $670 \mathrm{mV}$ & $605 \mathrm{mV}$ & $-10.7 \%$ \\
\hline
\end{tabular}


Table IV shows NVSRAM specific operation results at the array level. The nominal energy consumption of the NVSRAM is $2.5 \mathrm{~nJ}, 280 \mathrm{pJ}$ and $72 \mathrm{pJ}$ during FORMING, RESET and SET operations, respectively. Moreover, The nominal switching time is $5.6 \mathrm{~ns}, 8 \mathrm{~ns}$ and $500 \mathrm{~ns}$ for RESET, SET and FORMING, respectively.

TABLE IV. NVSRAM SPECIFIC OPERATIONS

\begin{tabular}{lc}
\hline \hline Nominal FORMING Energy & $2.5 \mathrm{~nJ}$ \\
Nominal SET Energy (STORE “1”) & $72 \mathrm{pJ}$ \\
Nominal RESET Energy (STORE “0”) & $172 \mathrm{pJ}$ \\
Nominal FORMING Switching Time & $500 \mathrm{~ns}$ \\
Nominal SET Switching Time & $8 \mathrm{~ns}$ \\
Nominal RESET Switching Time & $5.6 \mathrm{~ns}$ \\
\hline \hline
\end{tabular}

\section{IMPACT OF VARIABILITY ON NVSRAM METRICS}

In this section, $\mathrm{MC}$ simulations are performed to evalute the impact of OxRAM combined with CMOS variability on the NVSRAM performances. For the OxRAM, only actual variation are reported as the OxRAM model is calibrated on silicon. For the CMOS subsystem, on each simulation run, the $\mathrm{MC}$ analysis calculates every parameter randomly according to statistical distribution models. These statistical models are provided for active devices (MOSFETs) as well as for passive devices and cover corner cases. Fig. 22 shows the energy distributions resulting from $1000 \mathrm{MC}$ simulation runs after FORMING, RESET and SET operations. As expected, the energy consumption during FORMING is considerably high compared to SET and RESET operations. If we consider the distribution medians, the average energy consumptions are $2.5 \mathrm{~nJ}, 280 \mathrm{pJ}$ and $72 \mathrm{pJ}$ after FORMING, RESET and SET operations, respectively. High voltage programming pulses are required during FORMING and RESET, which leads to higher power consumption in both operations compared to SET. Note that a large energy is required during FORMING due to the large initial resistance of OxRAM cell which is in the pristine state. The voltage pulse level applied during FORMING is $5 \mathrm{~V}$ compared to 3.3V and 1.8V for RESET and SET operations, respectively. Hence, FORMING energy consumption is $8.9 \mathrm{x}$ and $34.7 \mathrm{x}$ higher than RESET and SET operations, respectively. Also, the energy consumption during RESET operation is $3.8 \mathrm{x}$ higher than that recorded during the SET operation.

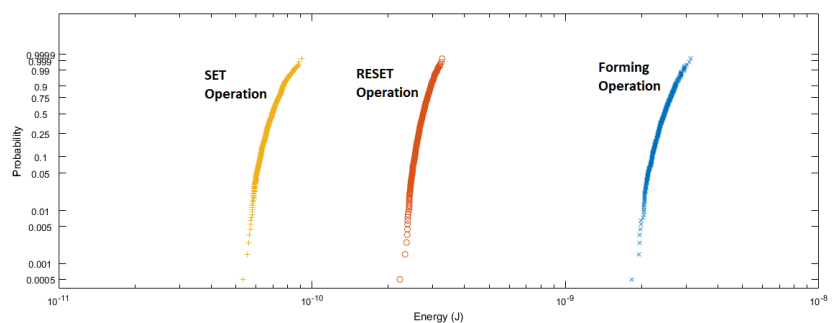

Fig. 22. MC simulations results for energy consumption during FORMING, SET and RESET operations
Fig. 23 shows the switching time distributions from $1000 \mathrm{MC}$ simulations runs after FORMING, RESET and SET operations. The switching time needed for the FORMING operation is significantly high compared to SET and RESET operations.

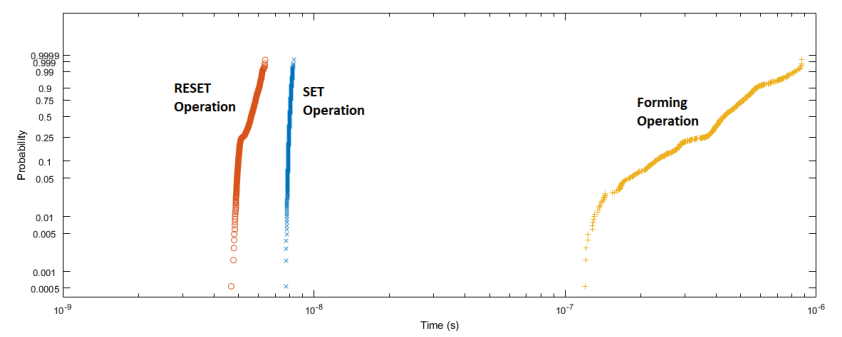

Fig. 23. MC simulations results for switching time during FORMING, SET and RESET operations

If we consider the distribution medians, the switching time average is $5.6 \mathrm{~ns}, 8 \mathrm{~ns}$ and $500 \mathrm{~ns}$ for RESET, SET and FORMING, respectively. Compared to SET (0.6 ns) and RESET (1.7 ns) switching time dispersion, larger programming pulses are required for FORMING operation in order to cover its large switching time dispersion (770 ns). It is worth noting that the magnitude of the voltage pulse direcltly affect the switching time of the NVSRAM STORE operation (SET and RESET). The higher the programming pulse, the shorter the switching time.

SNM stability criteria is also evaluated after $1000 \mathrm{MC}$ runs to assess the cell stability of the NVSRAM. Fig. 24 (a), (b), and (c) show simulated results of the 8T1R NVSRAM cell. HOLD, READ, and WRITE SNM (denoted by HSNM, RSNM, and WSNM, respectively) are considered. These plots present the best and worst SNM cases (highlighted in black) based on the $1000 \mathrm{MC}$ runs (gray lines). During the HOLD operation, HSNM varies between $560 \mathrm{mv}$ and 650 mv. During the READ operation, RSNM varies between 120 $\mathrm{mv}$ and $180 \mathrm{mv}$. Based on the butterfly curves of Fig. 24(c), it is observed that the process variations have negligible effect on the WSNM. In this regard, WSNM is equal to 790 $\mathrm{mv}$ in the worst case and $800 \mathrm{mv}$ in the best case. Table V summarizes SNM best and worst cases for HOLD, READ, and WRITE. Additionally, the success rate of data recovery is $100 \%$ as after the $1000 \mathrm{MC}$ runs, the proposed NVSRAM was able to restore the saved data.

As the main driving force when moving from SRAMs to NVSRAMs is the targeted application, the best and worst case SNM values are valuable information if the reliability criteria is considered. For instance, worst case SNMs can prevent the use of NVSRAMs in spatial or smartcard applications.

TABLE V. SNM BEST AND WORST CASES

\begin{tabular}{ccc}
\hline \hline & Best Case & Worst Case \\
\hline SNM (HOLD) & $650 \mathrm{mV}$ & $560 \mathrm{mV}$ \\
SNM (READ) & $180 \mathrm{mV}$ & $120 \mathrm{mV}$ \\
SNM (WRITE) & $800 \mathrm{mV}$ & $790 \mathrm{mV}$ \\
\hline \hline
\end{tabular}




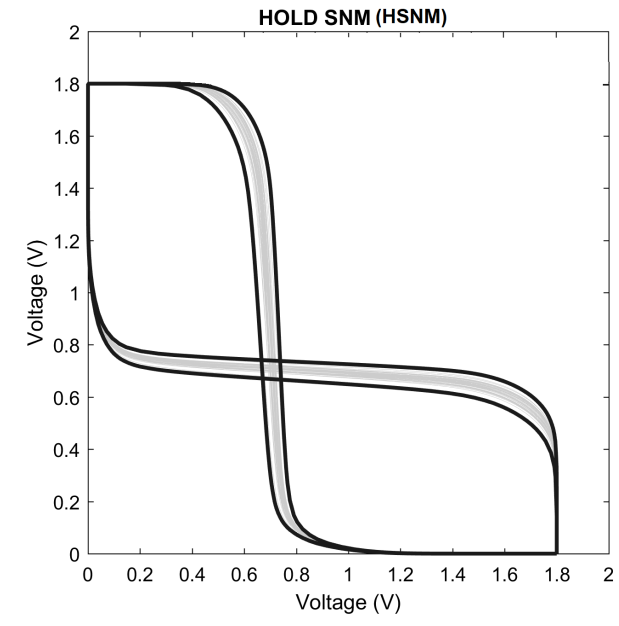

(a)

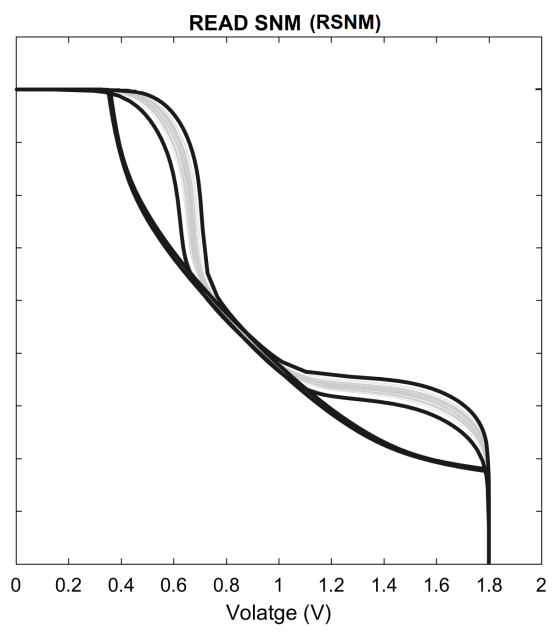

(b)

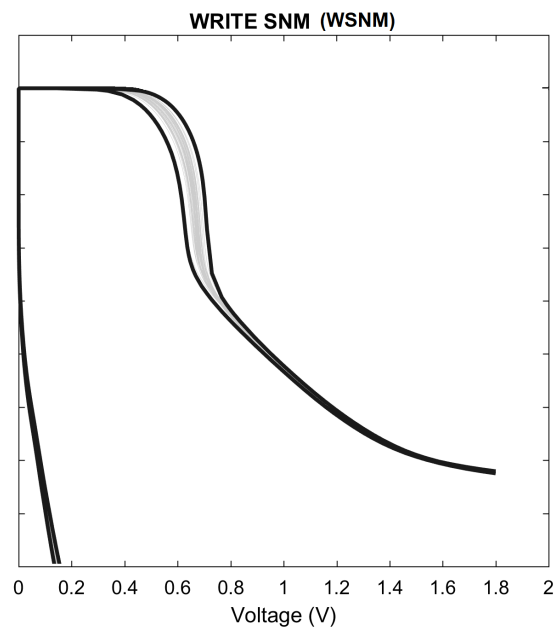

(c)

Fig. 24. (a) HOLD SNM (HSNM), (b) READ SNM (RSNM), and (c) WRITE SNM (WSNM)

\section{CONCLUSION}

This paper advances the state-of-the art of NVSRAM stability analysis by investigating the impact of OxRAM and CMOS variability on a novel 8T1R NVSRAM cell. The proposed 8T1R NVSRAM cell can be used in SRAM mode with classical HOLD, WRITE and READ operations. Also, this structure exhibits additional operations in non-volatile mode such as STORE and RESTORE. A comparison with a classical 6T SRAM is provided considering metrics such as area, energy consumption and SNMs. We demonstrated that a NVSRAM array is non-volatile but at the cost of larger area $(34.9 \%)$, and higher power consumption (2.6\%). This array can be kept in power OFF mode, which is useful for ultra-low power applications.

\section{ACKNOWLEDGMENT}

Authors would like to acknowledge the support of the Lebanese International University and Aix-Marseille University.

\section{Data Availability StATEMENT}

Data sharing not applicable to this article as no datasets were generated or analysed during the current study.

\section{REFERENCES}

[1] C. Dou et al., "Challenges of Emerging Memory and Memristor Based Circuits: Nonvolatile Logics, IoT Security, Deep Learning and Neuromorphic Computing." 2017 IEEE 12th International Conference on ASIC (ASICON), 2017.

[2] A. Banerjee, "Ultra-Low-Power Embedded SRAM Design for BatteryOperated and Energy-Harvested IoT Applications." Green Electronics, 2018.

[3] P. Singh et al., "Ultra-Low Power, Process-Tolerant 10T (PT10T) SRAM with Improved Read/Write Ability for Internet of Things (IoT) Applications." J. Low Power Electron. Appl. 2017, 7, 24.

[4] J.-M. Portal et al., "An Overview of Non-Volatile Flip-Flops Based on Emerging Memory Technologies." Journal of Electronic Science and Technology, vol. 12, no. 2, 2014, pp. 173-181.

[5] H. Bazzi et al., "Design of Hybrid CMOS Non-Volatile SRAM Cells in 130nm RRAM Technology." 2018 30th International Conference on Microelectronics (ICM), Sousse, Tunisia, 2018, pp. 228-231.
[6] Z. Abbas et al., "Impact of Technology Scaling on Leakage Power in Nano-Scale Bulk CMOS Digital Standard Cells." Microelectronics Journal, vol. 45, no. 2, 2014, pp. 179-195.

[7] J.-M. Portal et al., "Design and Simulation of a $128 \mathrm{~kb}$ Embedded Nonvolatile Memory Based on a Hybrid RRAM (HfO2 )/28 nm FDSOI CMOS Technology." IEEE Transactions on Nanotechnology, vol. 16, no. 4, pp. 677-686, 2017.

[8] A. Sheikholeslami, P. G. Gulak, "A survey of circuit innovation in ferroelectric random access memories." IEEE Proc., vol. 88, no5, pp. 667-689, May 2000.

[9] Y. Zhang et al., "Read Performance: The Newest Barrier in Scaled STT-RAM." in IEEE Transactions on Very Large Scale Integration (VLSI) Systems, vol. 23, no. 6, pp. 1170-1174, June 2015.

[10] G. W. Burr et al., "Recent Progress in Phase-Change Memory Technology." in IEEE Journal on Emerging and Selected Topics in Circuits and Systems, vol. 6, no. 2, pp. 146-162, June 2016.

[11] Y. Gonzalez-Velo et al., "TID Impact on Process Modified CBRAM Cells." 2015 15th European Conference on Radiation and Its Effects on Components and Systems (RADECS), Moscow, 2015, pp. 1-4.

[12] H. Aziza et al., "A lightweight write-assist scheme for reduced RRAM variability and power." Microelectronics Reliability 88 (2018): 6-10.

[13] J.S. Meena et al., "Overview of emerging nonvolatile memory technologies." Nanoscale Res Lett 9, 526 (2014).

[14] H. Bazzi et al. "Non-Volatile SRAM Memory Cells Based on ReRAM Technology." SN Applied Sciences, vol. 2, no. 9, 2020.

[15] H. Aziza et al., "Resistive RAMs as analog trimming elements." SolidState Electronics 142 (2018): 52-55.

[16] H. Bazzi et al., "RRAM-based non-volatile SRAM cell architectures for ultra-low-power applications." Analog Integr Circ Sig Process (2020).

[17] W.-X. You, et al., "A New 8T Hybrid Nonvolatile SRAM with Ferroelectric FET." IEEE Journal of The Electron Devices Society, vol 8, 2020, pp. 171-175.

[18] M. Kobayashi, et al., "A Nonvolatile SRAM Integrated with Ferroelectric HfO2 Capacitor for Normally-off and Ultralow Power IoT Application." 2017 Symposium on VLSI Technology, 2017.

[19] S. Majumdar et al., "Hybrid CMOS-OxRAM based 4T-2R NVSRAM with efficient programming scheme." 2016 16twh Non-Volatile Memory Technology Symposium (NVMTS), Pittsburgh, PA, 2016, pp. $1-4$.

[20] W. Wei et al., "Design of a Nonvolatile 7T1R SRAM Cell for Instanton Operation." in IEEE Transactions on Nanotechnology, vol. 13, no. 5, pp. 905-916, Sept. 2014.

[21] M.-F. Chang et al., "Challenges at Circuit Designs for Resistive-Type Nonvolatile Memory and Nonvolatile Logics in Mobile and Cloud Applications." 2014 12th IEEE International Conference on SolidState and Integrated Circuit Technology (ICSICT), 2014. 
[22] S. Shreya et al., "Differential Spin Hall Effect-Based Nonvolatile Static Random Access Memory for Energy-Efficient and Fast Data Restoration Application." in IEEE Transactions on Magnetics, vol. 55, no. 9, pp. 1-11, Sept. 2019.

[23] W. Kang et al., "Low Store Power, High Speed, High Density, Nonvolatile SRAM Design With Spin Hall Effect-Driven Magnetic Tunnel Junctions." IEEE Transactions on Nanotechnology, 2016, pp. $1-1$

[24] X. Li et al., "Design of Nonvolatile SRAM with Ferroelectric FETs for Energy-Efficient Backup and Restore." IEEE Transactions on Electron Devices, vol. 64, no. 7, 2017, pp. 3037-3040.

[25] K. Huang, et al., "A Low Active Leakage and High Reliability Phase Change Memory (PCM) Based Non-Volatile FPGA Storage Element." IEEE Transactions on Circuits and Systems I: Regular Papers, vol. 61, no. 9, 2014, pp. 2605-2613.

[26] S.K. Kingra et al., "Stability Analysis of Hybrid CMOS-RRAM Based 4T-2R NVSRAM." 2017 15th IEEE International New Circuits and Systems Conference (NEWCAS), 2017.

[27] M. Bocquet et al., "Compact modeling solutions for OxRAM memories." 2013 IEEE Faible Tension Faible Consommation, Paris, 2013, pp. 1-4.

[28] M. Bocquet et al., "Robust Compact Model for Bipolar Oxide-Based Resistive Switching Memories." IEEE Transactions on Electron Devices, vol. 61, no. 3, 2014, pp. 674-681.

[29] Y. Joly et al., "Impact of hump effect on MOSFET mismatch in the sub-threshold area for low power analog applications." International Conference on Solid-State and Integrated Circuit Technology, 2010.

[30] Y. Joly et al., "Matching degradation of threshold voltage and gate voltage of NMOSFET after Hot Carrier Injection stress." microelectronics reliability 51.9-11 (2011): 1561-1563.

[31] Y. Bai et al., "Study of Multi-Level Characteristics for 3D Vertical Resistive Switching Memory." Scientific Reports, vol. 4, no. 1, 2014.

[32] H. Aziza et al., "Evaluation of OxRAM cell variability impact on memory performances through electrical simulations." Non-Volatile Memory Technology Symposium Proceeding, 2011.

[33] H. Bazzi et al., "Novel RRAM CMOS Non-Volatile Memory Cells in 130nm Technology." ICCA, Beirut, 2018, pp. 390-393.

[34] A. Benoist et al., "28nmadvancedCMOS resistive RAM solution as embedded non-volatile memory." In 2014 IEEE International Reliability Physics Symposium, pages 2E.6.1-2E.6.5, June 2014.

[35] F. Nardi et al., "Resistive Switching by Voltage-Driven Ion Migration in Bipolar RRAM Part I : Experimental Study." IEEE Transactions on Electron Devices, vol. 59, no. 9, pp. 2461-2467, 2012.

[36] B. Hajri, et al., "RRAM Device Models: A Comparative Analysis With Experimental Validation." IEEE Access 7 (2019): 168963-168980.

[37] B. Hajri et al., "Oxide-based RRAM models for circuit designers: A comparative analysis." International Conference on Design \& Technology of Integrated Systems In Nanoscale Era (DTIS). IEEE, 2017.

[38] T. Cabout et al., "Temperature impact (up to 200oC) on performance and reliability of HfO2-based RRAMs." in Proc. IEEE International Memory Workshop, no. V, 2013, pp. 4-7.

[39] T. Diokh et al., "Investigation of the impact of the oxide thickness and RESET conditions on disturb in HfO2-RRAM integrated in a $65 \mathrm{~nm}$ CMOS technology." in Proc. IEEE International Reliability Physics Symposium, 2013, pp. 3-6.

[40] A. Fantini et al., "Intrinsic switching variability in HfO2 RRAM." in Proc. IMW, Monterey, CA, 2013, pp. 30-33.

[41] http://iterature.cdn.keysight.com/litweb/pdf/B1500-90090.pdf

[42] A. Mazreah et al., "A Low Power SRAM Base on Novel Word-Line Decoding." World Academy of Science, 2008.

[43] O. Turkyilmaz et al., "RRAM-based FPGA for normally off, instantly on applications." 2012 IEEE/ACM International Symposium on Nanoscale Architectures, Amsterdam, 2012, pp. 101-108.

[44] S. Sheu et al., "A ReRAM integrated 7T2R non-volatile SRAM for normally-off computing application." 2013 IEEE Asian Solid-State Circuits Conference (A-SSCC), Singapore, 2013, pp. 245-248.

[45] P. Chiu et al., "Low Store Energy, Low VDDmin, 8T2R Nonvolatile Latch and SRAM With Vertical-Stacked Resistive Memory (Memristor) Devices for Low Power Mobile Applications." in IEEE Journal of Solid-State Circuits, vol. 47, no. 6, pp. 1483-1496, 2012.
[46] A. Gurjar et al., "An Analytical Approach to Design VLSI Implementation of Low Power, High Speed SRAM Cell Using Submicron Technology." International Journal of VLSI design \& Communication Systems (VLSICS), 2011. 2(4): p. 143-153.

[47] Ishibashi, Koichiro, and Kenichi Osada. "Low Power and Reliable SRAM Memory Cell and Array Design." Springer, 2011.

[48] E. Seevinck, F. J. List, J. Lohstroh, "Static-Noise Margin Analysis of MOS SRAM cells." IEEE Journal of Solid-State Circuits, Volume 22, pp. 748-754, 1987. 